\title{
Establishing Lot Size through Sanitation Clean Breaks in Produce Packing Facilities ${ }^{1}$
}

\author{
B. Chapman and M.D. Danyluk ${ }^{2}$
}

\section{Why Have Clean Breaks?}

Many produce packers establish lots to trace their products and to limit risk to their business if a food safety contamination event occurs. Whether a packer determines a lot by date, grower, field, buyer, or some other means, a sanitation "clean break" is needed before and after the production of that lot to consider it separate from other production. In a number of recent produce-related food safety events, the lack of a defined clean break resulted in a recall that covered the entire production season. This publication highlights the importance of sanitation clean breaks in produce packing facilities and identifies what is needed to establish clean breaks in these facilities.

\section{What Is a Sanitation Clean Break?}

A sanitation clean break, commonly referred to as a "clean break," is a defined production break that involves a documented, verified, and validated cleaning and sanitation process of all food contact surfaces. Under the current proposed produce safety rule, food contact surfaces are defined as "...those surfaces that contact human food and those surfaces from which drainage, or other transfer, onto the food or onto surfaces that contact the food ordinarily occurs during the normal course of operations" (78 Federal Register 2013). These surfaces include the food contact surfaces of equipment and tools used during harvest, packing, and holding.

\section{What Is the Difference Between Cleaning and Sanitizing?}

Cleaning is commonly defined as the removal of physical soil or debris from a surface. In a packing facility, soil may include, but is not limited to, plant debris, earth, mud, dust, rust or other mineral deposits, wax buildup, PLU stickers or adhesives, and biofilms.

Sanitizing is commonly defined as the reduction of microorganisms on a cleaned surface. Unless a surface is clean, it is extremely difficult to sanitize. Soil remaining on the surface may interact with the sanitizing chemical, which can prevent close contact between the surface and the sanitizer or inactivate the sanitizing agent.

\section{Establishing a Clean Break}

The recommendations below are drawn from Good Agricultural Practices (GAPs), Best Management Practices (BMPs), Good Manufacturing Practices (GMPs), and the scientific literature.

All food contact surfaces need to be constructed so they can be cleaned and sanitized (these surfaces are often described as Zone 1 in food processing and manufacturing).

Sanitation standard operating procedures (SSOPs) for cleaning and sanitizing need to be in place at a facility and

1. This document is FSHN13-10, one of a series of the Food Science and Human Nutrition Department, UF/IFAS Extension. Original publication date August 2013. Visit the EDIS website at http://edis.ifas.ufl.edu.

2. Benjamin Chapman, assistant professor, Department of 4-H Youth Development and Family \& Consumer Sciences, North Carolina State University, Raleigh, NC 27695; Michelle D. Danyluk, associate professor, Food Science and Human Nutrition Department, Citrus Research and Education Center, Lake Alfred, FL 33850. 
specifically designed for that facility. These SSOPs should include the following information:

- Identify the exact surfaces/areas being cleaned and sanitized and what areas the packer defines as a food contact surface. Provide the specific protocols for how the different surfaces will be cleaned.

- Specify the frequency at which each procedure is being conducted, and identify the employee responsible for the procedure.

- Evaluate the condition of the food contact surfaces to ensure that they can be cleaned and sanitized.

- Identify compounds used and how they are used. This may include concentration, mixing procedure, water source, solution $\mathrm{pH}$, contact time, temperature, and rinse procedure (if applicable).

All compounds used with the objective of cleaning and sanitizing food contact surfaces should be labeled as such; they also must be used according to the manufacturer's directions and validated as suitable for their use. Records that support the SSOPs must be completed and available. Records should include when the cleaning and sanitizing activity occurred (date and time), the compound concentration used, and the individual(s) who carried out the tasks.

If there are any issues, corrective action records must be kept. These must indicate the steps taken to restore sanitary conditions, the efforts to rework or divert any product that may have been processed under unsanitary conditions, and the actions taken to prevent this issue from occurring in the future.

\section{Summary}

A clean break is needed between groups of products for food protection regulators to consider produce as separate from other produce packed off the same line. Determining a clean break is important to limit the scope of a recall. Packers can determine lot size based on what is practical and the amount of risk that their business is comfortable with. Food protection regulators define lot size as when a clean break occurs before and after a group of products. For instance, if a packer chooses to have a daily documented and verified clean break, the packer would establish one lot per day, as defined by food protection regulators. In other situations, a packer may choose to have a clean break conducted weekly, meaning that a food safety incident could result in a week's worth of production being recalled.

\section{Quick Facts about Clean Breaks}

- To limit the scope of a potential food safety event, food contact surfaces need to be regularly cleaned and sanitized.

- Procedures for cleaning and sanitation should be documented and include location, frequency, and chemicals used.

- Chemical compounds for cleaning and sanitation should be validated for their intended use and used following manufacturer's directions.

- Documenting when, how, and why cleaning and sanitation occurred is necessary to establish a lot.

\section{References}

"Current Good Manufacturing Practice and Hazard Analysis and Risk-Based Preventive Controls for Human Food." 78 Federal Register 11 (16 January 2013). http://www.gpo. gov/fdsys/pkg/FR-2013-01-16/html/2013-00125.htm. 